\title{
Methodology Report Hunting for Serine 276-Phosphorylated p65
}

\section{Anneleen Spooren, Krzysztof Kolmus, Linda Vermeulen, Karlien Van Wesemael, Guy Haegeman, and Sarah Gerlo}

\author{
Laboratory of Eukaryotic Gene Expression (LEGEST), Department of Physiology, Ghent University, K.L. Ledeganckstraat 35, \\ 9000 Ghent, Belgium
}

Correspondence should be addressed to Sarah Gerlo, sarah.gerlo@ugent.be

Received 13 August 2009; Revised 11 November 2009; Accepted 17 November 2009

Academic Editor: Susan A. Rotenberg

Copyright (C 2010 Anneleen Spooren et al. This is an open access article distributed under the Creative Commons Attribution License, which permits unrestricted use, distribution, and reproduction in any medium, provided the original work is properly cited.

\begin{abstract}
The transcription factor nuclear factor kappaB (NF- $\kappa \mathrm{B})$ is one of the central mediators of inflammatory gene expression. Several posttranslational modifications of NF- $\kappa \mathrm{B}$, regulating its transactivation ability, have been described. Especially phosphorylation of the NF- $\kappa$ B subunit p 65 has been investigated in depth and several commercial phosphospecific antibodies, targeting selected p65 residues, are available. One of the p65 residues, that is subject to phosphorylation by protein kinase A (PKA) as well as by mitogenstimulated kinase-1 (MSK-1), is the serine at position 276. Here, we have performed a detailed analysis of the performance of the most commonly used commercial anti-P-p65 Ser276 antibodies. Our findings indicate that at least three widely used anti-P-p65 Ser276 antibodies do not detect p65 in vivo via Western Blot, but instead crossreact with PKA-regulated proteins. As PKA is one of the main kinases responsible for phosphorylation of p65 at Ser276, this observation warrants cautious interpretation of data generated using the tested antibodies.
\end{abstract}

\section{Introduction}

The transcription factor nuclear factor kappaB (NF- $\kappa \mathrm{B})$ was originally identified as the factor driving immunoglobulin transcription in B-cells [1]. Since then evidence has accumulated, identifying NF- $\kappa \mathrm{B}$ as the master regulator of inflammatory gene expression in a variety of cell types $[2,3]$. The NF- $\kappa \mathrm{B}$ family of transcription factors consists of a group of proteins (p50/p105, p52/p100, p65 (relA), c-Rel, and RelB), all of which contain the N-terminal Rel-homology domain (RHD), that is responsible for their DNA-binding and homo- or heterodimerization ability. In addition, p65, c-Rel and RelB also contain a transactivation domain (TAD). The NF- $\kappa \mathrm{B}$ proteins are kept in the cytoplasm through physical interaction with inhibitors of the $\mathrm{I} \kappa \mathrm{B}$ (inhibitor of $\kappa \mathrm{B})$ family. In response to proinflammatory cytokines or pathogen-associated molecular patterns, the I $\kappa \mathrm{B}$ kinase (IKK), an upstream kinase complex, is activated. Activated IKK phosphorylates the I $\kappa \mathrm{B}$ molecules, targeting them for ubiquitination-dependent degradation, and allowing the migration of NF- $\kappa \mathrm{B}$ dimers to the nucleus, where they can activate gene transcription. More recent studies have shown that, in many instances, I $\kappa$ B degradation and consequent NF$\kappa \mathrm{B}$ nuclear translocation are not sufficient for full-blown NF$\kappa \mathrm{B}$-dependent gene expression. A multitude of posttranslational modifications of the NF- $\kappa$ B subunits, affecting their transactivation ability, has indeed been described [4-7].

The prototypical, most studied, NF- $\kappa$ B complex is the p50-p65 dimer, and especially posttranslational modifications of the $\mathrm{p} 65$ subunit have been investigated in great detail. The p65 residue contains several serine residues, which have been shown to be subject to phosphorylation $[5,8]$. One of the p65 serine residues, which was originally shown to be phosphorylated by the protein kinase A (PKA) upon lipopolysaccharide (LPS) triggering, is the serine at position 276 [9]. Later it was discovered that the p65 serine 276 (Ser276) residue is also a target of the mitogen- and stressactivated kinase-1 (MSK-1) after TNF- $\alpha$ stimulation [10]. Since its original demonstration in 1997, the role of phosphorylation of the p65 Ser276 residue has been intensively 
studied, and the phosphorylation has been shown to be involved in the expression of a variety of NF- $\kappa \mathrm{B}$-dependent genes, in different cell types and upon different stimuli. Most studies agree that Ser276 phosphorylation is required for the recruitment of the transcriptional coactivator CBP (CREBbinding protein) and, hence, activates transcription of particular p65 target genes $[10,11]$. Very recently, a "knockin" mouse, expressing p65 with the Ser276 mutated to alanine was generated [12]. Unfortunately these mice showed a variegated phenotype, making it difficult to interpret the physiological function of $\mathrm{p} 65$ Ser276 phosphorylation.

Most of the reports investigating the function of p65 Ser276 phosphorylation have made use of a commercial antibody (Cell Signaling no. 3037), directed against the phosphorylated p65 Ser276 residue (for a complete list of publications in which anti-phospho-p65 Ser276 antibodies were used, we refer toSupplementary Table 1 in Supplementary Material available online at doi:10.1155/2009/275892). This antibody was generated using a synthetic Keyhole limpet hemocyanin (KLH)-coupled phosphopeptide, corresponding to the amino acid residues surrounding Ser276 of human NF- $\kappa$ B p 65.

In this study, we have summarized our experimental data obtained with the Cell Signaling no. 3037 and three other commonly used anti-phospho(P)-p65 Ser276 antibodies. Our findings suggest these antibodies are not suitable for detection of Ser276-phosphorylated p65 in vivo by Western Blotting and indicate that studies into the function of $\mathrm{p} 65$ Ser276 phosphorylation should be supported by additional, antibody-independent assays.

\section{Materials and Methods}

2.1. Reagents. Isoproterenol (iso), forskolin, phorbol 12 -myristate 13-acetate (PMA), chloro-phenyl-thio-cAMP (cAMP), H89, and lipopolysaccharide (LPS) were purchased from Sigma-Aldrich (St. Louis, MO). Recombinant human tumor necrosis factor- $\alpha$ (TNF- $\alpha$ ) was obtained from the Department of Molecular Biology of Gent University (DMBR, Gent, Belgium). siPKAc $\alpha$ (M-004649-01), sip65 (M-00353302), sip105/p50 (M-003520-01), and si-c-Rel (M-00476801) SMART pools and siControl (D-001210-05) were from Dharmacon (Lafayette, CO).

2.2. Primary Antibodies. Four rabbit polyclonal antibodies directed against the phosphorylated Ser276 residue of p65 were used in this study: anti-P-p65 Ser276 (no. 3037) from Cell Signaling (Danvers, MA), anti-P-p65 Ser276 (no. 11011) from Signalway Antibody (SAB, Pearland, TX), anti-P-p65 Ser276 (no. 100-401-264) from Rockland (Gilbertsville, PA), and a homemade anti-P-p65 Ser276 antibody (kind gift of Prof. Dr. P. Cohen, University of Dundee, UK; produced and purified by Drs. C. Armstrong and J. Leitch). Anti-p65 (C-20), anti-CBP, anti-p105/p50, anti-c-Rel, and anti-PKAc $\alpha$ were from Santa Cruz (Santa Cruz, CA). Anti-tubulin was from Sigma-Aldrich (St. Louis, MO).
2.3. Cell Culture. L929sA mouse fibroblasts were described previously [13]. Raw264.7 mouse macrophages, C2C12 mouse myoblasts, and L363 human multiple myeloma cells were obtained from the European Collection of Cell Cultures (Salisbury, UK). The human astrocytoma cell line 1321N1 was a kind gift from Prof. Dr. Müller (University of Bonn, Germany). NF- $\kappa$ B p65-deficient, and wild type p65 (wt p65) or p65 Serine to alanine mutated (p65 S/A) reconstituted, mouse embryonic fibroblasts (MEFs) were a gift from Dr. Okazaki (Juntendo University, Japan). L363, Raw264.7, and C2C12 cells were maintained in RPMI-1640 with GlutamaxI, supplemented with $10 \%$ FCS, $100 \mathrm{U} / \mathrm{mL}$ penicillin, and $100 \mu \mathrm{g} / \mathrm{mL}$ streptomycin. All other cells were cultured in DMEM with $10 \%$ FCS, $100 \mathrm{U} / \mathrm{mL}$ penicillin and $100 \mu \mathrm{g} / \mathrm{mL}$ streptomycin. Cells were maintained at $37^{\circ} \mathrm{C}$ in a humidified $5 \% \mathrm{CO}_{2}$ atmosphere. Adherent cells were passaged using $0,05 \%(\mathrm{w} / \mathrm{v})$ of trypsin in $0,4 \%(\mathrm{w} / \mathrm{v})$ EDTA (all cell culture reagents were from Invitrogen, Carlsbad, CA).

2.4. Western Blotting. Total cellular extracts were made in SDS sample buffer $(62,5 \mathrm{mM}$ Tris-HCl pH 6.8, $2 \%$ SDS, $10 \%$ glycerol, $0,5 \% \beta$-mercapto-ethanol). Equal amounts of total lysates from each condition were resolved by $8,5 \%$ SDSPAGE, transferred onto nitrocellulose membranes and analysed by Western Blotting. Chemiluminescent detection was performed using horse-radish peroxidase (HRP)-coupled secondary antibodies and Western lightning chemiluminescent reagent plus (Perkin Elmer LifeSciences, Boston, MA) on the Kodak image station 440CF. For applications that required higher sensitivity, Immobilion Nitrocellulose was used, in combination with fluorescent secondary antibodies (Rockland, Gilbertsville, PA). Detection was performed on the Odyssey Imaging System (Licor, Lincoln, NA). For preadsorption of the antibodies in blocking experiments, twice the volume of P-p65 Ser276 blocking peptide (Cell Signaling, no. 1437) as volume of P-p65 Ser276 antibody was combined and incubated for 30 minutes at $4^{\circ} \mathrm{C}$, prior to adding the antibody/peptide mixture to the blots.

2.5. siRNA Silencing of $p 65$ and PKAca. For siRNA experiments cells were seeded in 6 wells (200000 cells/well) and transfected using the calcium phosphate method with $50 \mathrm{nM}$ siGENOME SMART pools. siControl-transfected samples were used to assess aspecific effects. 48 hours after transfection and after overnight starvation, cells were induced for the indicated time periods. Subsequently, cells were lysed in SDS sample buffer for Western Blotting analysis.

2.6. In Vitro Kinase Assay. Recombinant GST-p65 $5^{12-317}$ and the S276C-mutated version were produced and purified according to the manufacturer's instructions (Amersham Pharmacia Biotech, Rainham, UK). $2 \mu \mathrm{M}$ of the protein was incubated at $30^{\circ} \mathrm{C}$ for $20 \mathrm{~min}$ in kinase buffer $(50 \mathrm{mM}$ Tris $\mathrm{pH} 7.4,10 \mathrm{mM} \mathrm{Mg}(\mathrm{Ac})_{2}, 0.1 \mathrm{mM}$ EGTA, and $0.1 \% \beta$ mercaptoethanol), supplemented with $0.1 \mathrm{mM}$ ATP and $15 \mathrm{ng}$ of recombinant active MSK-1 (Upstate, Billerica, MA). $250 \mathrm{ng}$ of recombinant proteins were subsequently spotted 


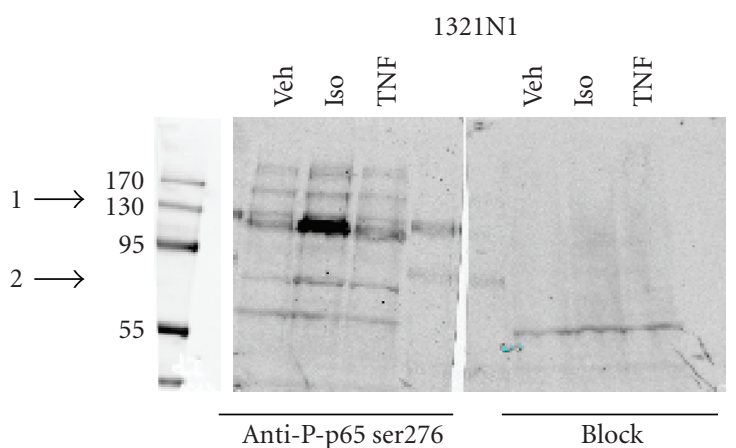

(a)

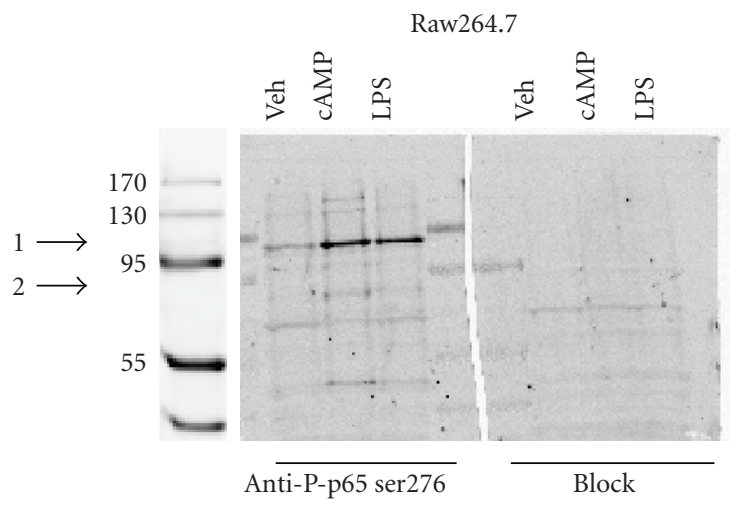

(c)

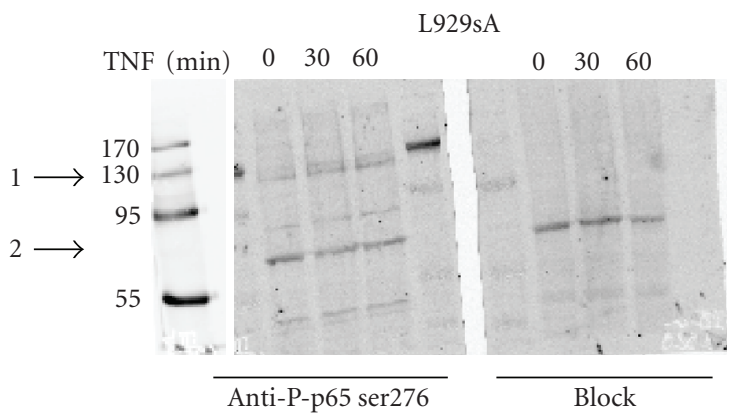

(b)

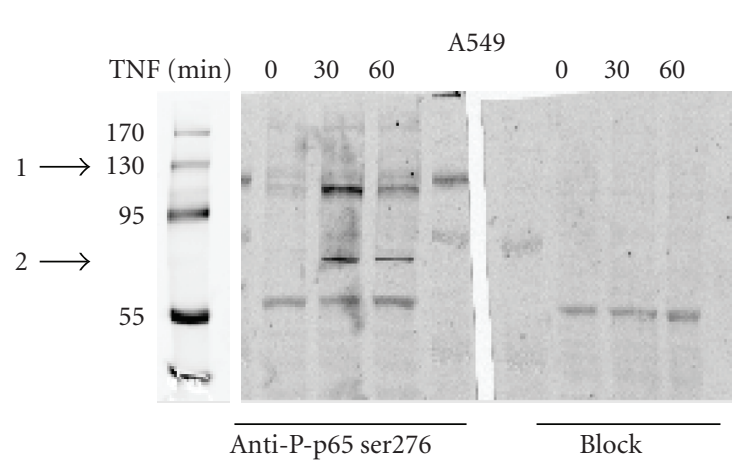

(d)

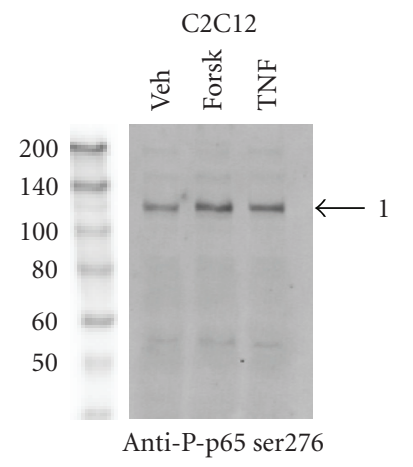

(e)

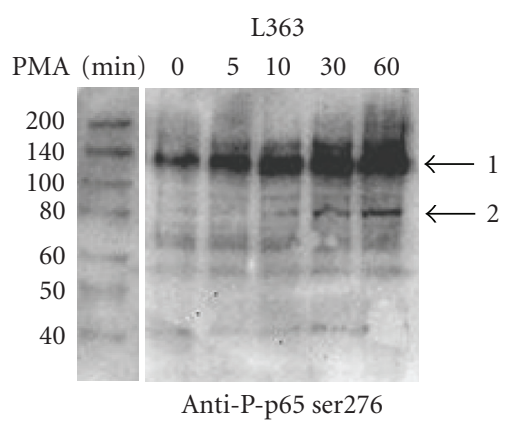

(f)

Figure 1: Anti-phospho-p65 Ser276 (Cell Signaling no. 3037) detects atypical (with MWs of 80 and $130 \mathrm{kDa}$ ), inducible immunoreactivities in different cell types, induced with different NF- $\kappa B$-activating agents. Cells were stimulated for increasing times (L929sA, A549, L363) or for $30 \mathrm{~min}$ (Raw264.7, C2C12, 1321N1) with TNF- $\alpha(2000 \mathrm{IU} / \mathrm{mL})$, LPS $(1 \mu \mathrm{g} / \mathrm{mL})$, PMA $(10 \mathrm{ng} / \mathrm{mL})$, forskolin (forsk, $10 \mu \mathrm{M})$ or isoproterenol (iso, $10 \mu \mathrm{M}$ ). For blocking experiments, lysates were loaded on one gel in duplicate and after transfer, blots were cut in half. Immunodetection was performed in parallel, using Cell Signaling no. 3037 or Cell Signaling no. 3037 preincubated for at least 30 minutes with double volume of blocking peptide (BLOCK). Arrow 1 indicates an immunoreactive band with an MW of $130 \mathrm{kDa}$; arrow 2 indicates an immunoreactive band of $80 \mathrm{kDa}$.

on nitrocellulose membrane, or separated via SDS-PAGE, and subjected to Western Blotting.

\section{Results}

3.1. Anti-Phospho-p65 Ser276 (Cell Signaling no. 3037) Detects a Prominent $130 \mathrm{kDa}$ Band, and a Weak $80 \mathrm{kDa}$ Band, Induced by Different Stimuli (LPS, PMA, TNF, cAMP, Forskolin, Isoproterenol) in Different Cell Types. Both Bands Can Be Blocked with the Phosphopeptide Used for Immunization. The anti-phospho-p65 Ser276 (no. 3037), distributed by Cell Signaling (Danvers, MA), was generated in rabbits using a synthetic, KLH-coupled phosphopeptide, corresponding to 


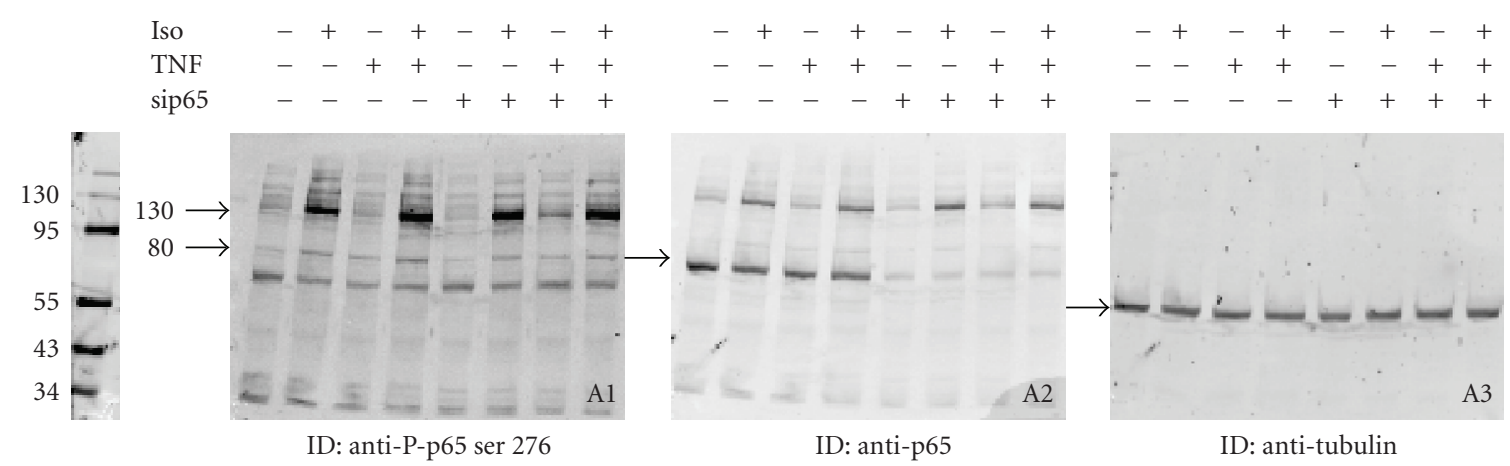

(a)

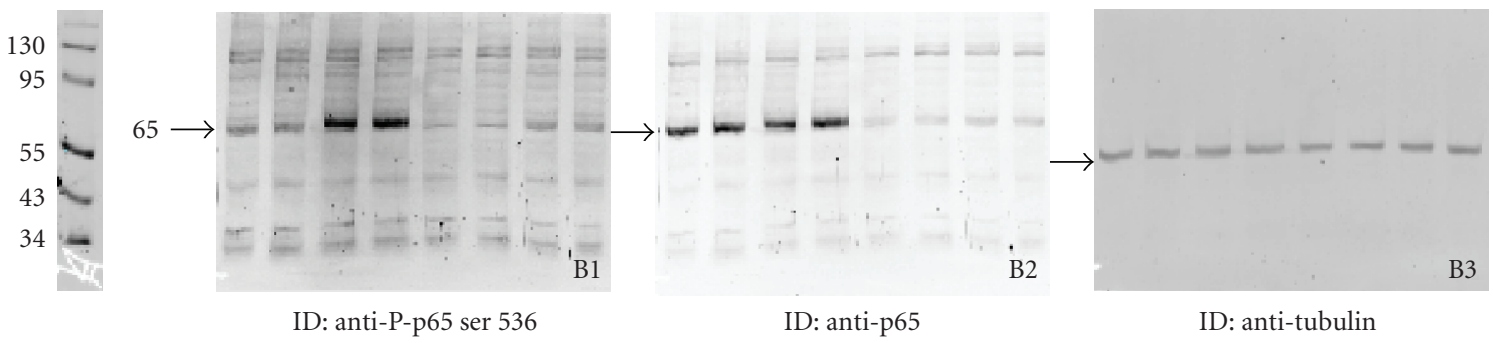

(b)

Figure 2: The Cell Signaling no. 3037 anti-P-p65 Ser276-immunoreactive band persists in cells where p65 is silenced via an siRNA approach. $1321 \mathrm{~N} 1$ cells were transiently transfected with control or p65 siRNA as described in Section 2. Cells were induced for 90 min with TNF- $\alpha$ (2000 IU/mL), iso $(10 \mu \mathrm{M})$, or a combination of both stimuli. Presence of Ser276-phosphorylated p65 in cell lysates was analysed by Western Blot using Cell Signaling no. 3037 (A1). 130 and 80 kDa immunoreactivities are indicated by arrows. Western Blot to detect anti-P-p65Ser536 (65 kDa arrow) was performed on the same lysates (B1). Blots A1 and B1 were extensively washed and reprobed with anti-p65 to investigate efficiency of p65 knock-down. Anti-p65 detected bands are marked with arrows (A2, B2). Finally, blots were reprobed with anti-tubulin to check loading efficiency. Anti-tubulin-detected bands are marked with arrows (A3, B3).

the residues surrounding Ser276 of human p65. According to the accompanying data sheet, the antibody only detects p65 when it is phosphorylated at the Ser276 residue. Additional information supplied by the datasheet is that, whereas it was generated using a human peptide, the antibody crossreacts with mouse and rat (and possibly dog) P-Ser276 p65. Also according to the data sheet, Ser276-phosphorylated p65 migrates at $80 \mathrm{kDa}$, instead of the expected $65 \mathrm{kDa}$, in reducing gels. As shown in Figure 1, we detected several immunoreactive bands in lysates from different human (L363, 1321N1, A549) and mouse (L929sA, Raw264.7, $\mathrm{C} 2 \mathrm{C} 12)$ cell types. Phosphorylation of p65 at Ser276 has been described after cAMP-independent activation of PKA by LPS [9], as well as after cAMP-dependent stimulation of PKA by the adenylyl cyclase activator forskolin [14]. In addition, p65 was phosphorylated at Ser276 by MSK-1 upon TNF- $\alpha$ treatment [10]. We indeed observed that signals activating PKA (LPS, cAMP, isoproterenol and forskolin) or MSK1 (TNF, PMA) induced immunoreactive bands recognized by the Cell Signaling no. 3037 antibody. In all investigated cell types, the major immunoreactive band, induced by all investigated stimuli, migrated with an apparent molecular weight (MW) of $130 \mathrm{kDa}$ (arrow 1). In most cell types, a minor $80 \mathrm{kDa}$ band (arrow 2), as expected based upon the information supplied by the Cell Signaling datasheet, was also induced. To investigate whether these immunoreactive bands represent specific interactions with sequences homologous to that of the phosphopeptide used for immunization, we performed blocking experiments in which the anti-Pp65 Ser276 antibody was preadsorbed with this phosphopeptide. As demonstrated in Figure 1, both the 80 and $130 \mathrm{kDa}$ induced immunoreactivities disappeared when the blots were incubated with preadsorbed anti-P-p65 Ser276 antibody, indicating that the immunoreactivity is indeed the result of a specific interaction with an amino acid sequence homologous to that of the immunizing phosphopeptide.

\subsection{The Cell Signaling no. 3037-Detected Bands Are Not Abro-} gated upon siRNA Mediated Knock-Down of p65. Because we observed, unexpectedly, that cAMP-activating signals, such as isoproterenol or forskolin, in addition to the $80 \mathrm{kDa}$ band described in the Cell Signaling no. 3037 datasheet, potently induced a $130 \mathrm{kDa}$ band that was recognized in a specific manner by the Cell Signaling no. 3037 antibody in different cell types, we further investigated the authenticity of this band using an siRNA approach in the 1321N1 human astrocytoma. As shown in Figure 2, whereas our siRNA approach led to efficient knock-down of p65 (A2, $\mathrm{B} 2)$, the $130 \mathrm{kDa}$, isoproterenol-induced immunoreactivity 


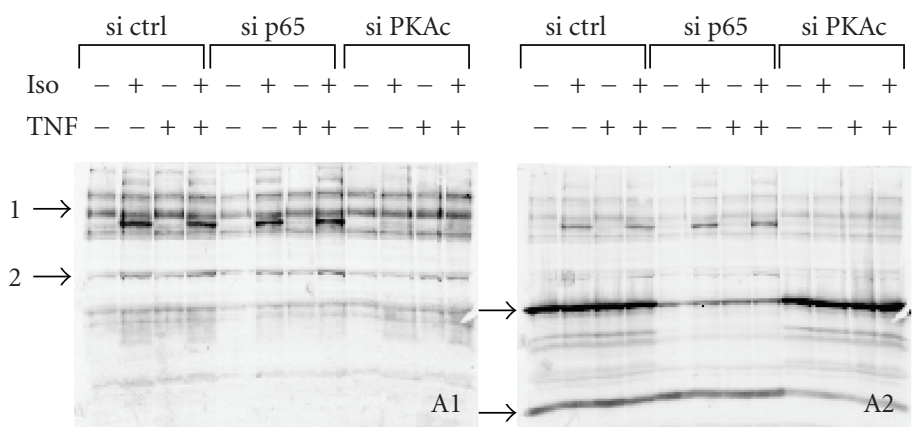

ID: anti-P-p65 ser276 (SAB) ID: anti-p65+anti-PKAc
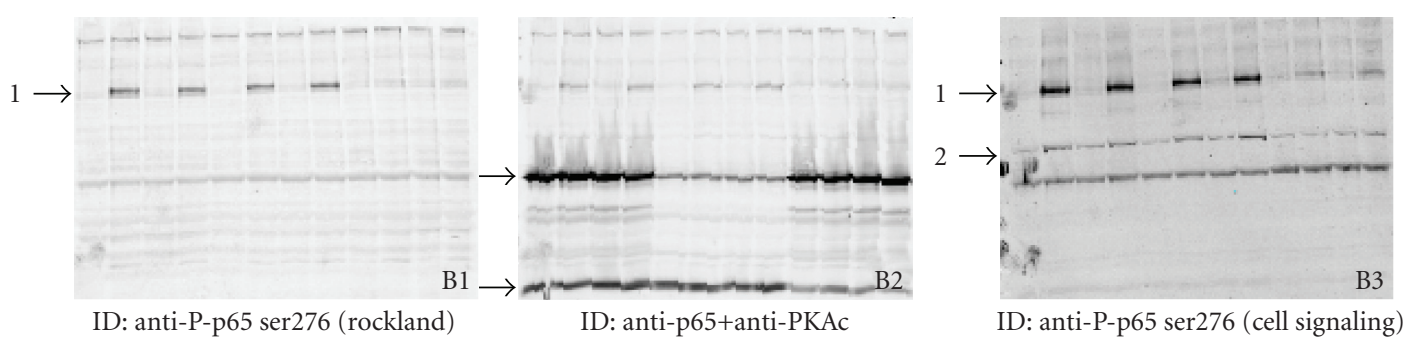

(a)

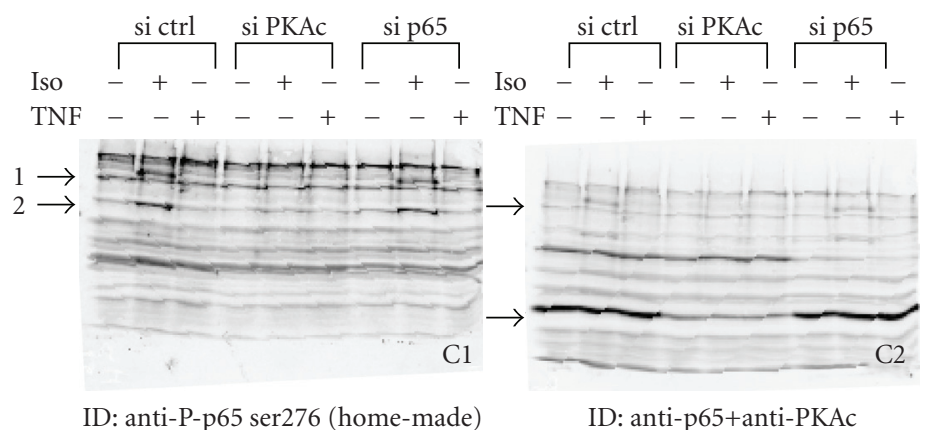

(b)

Figure 3: Four independent anti-P-p65 Ser276 antibodies detect inducible bands, with MWs of 80 and $130 \mathrm{kDa}$, that do not disappear upon p65 knock-down, but are inhibited when PKAc $\alpha$ is silenced. $1321 \mathrm{~N} 1$ cells were transiently transfected with control, p65 or PKAc $\alpha$ siRNA, as described in Section 2. Cells were induced for $90 \mathrm{~min}$ with TNF- $\alpha(2000 \mathrm{IU} / \mathrm{mL})$, iso $(10 \mu \mathrm{M})$ or a combination of both stimuli. Presence of Ser276-phosphorylated p65 in cell lysates was analysed by Western Blot using SAB no. 11011 (A1), Rockland no. 100-401-264 (B1), Cell Signaling no. 3037 (B3), or a homemade anti-P-p65 Ser276 antibody (C1). 130 and $80 \mathrm{kDa}$ immunoreactivities are indicated by arrows (1 and 2, resp.). Blots were extensively washed and reprobed with a mixture of anti-p65 and anti-PKAc $\alpha$ to investigate knock-down efficiency. Anti-p65-detected bands are marked by the upper arrow, anti-PKAc $\alpha$-reactive bands are marked by the lower arrow (A2, B2, C2). Tubulin loading controls are added as Supplementary Figure 1.

detected by Cell Signaling no. 3037, did not disappear or even diminish (A1). Moreover, the $80 \mathrm{kDa}$, presumable PSer276 p65, band, induced by isoproterenol and, to a lesser extent, TNF- $\alpha$, also remained equally apparent in cells were p65 expression was silenced (Figure 2, A1). As a control, we also investigated whether $\mathrm{p} 65$ knock-down abrogated TNF$\alpha$-induced phosphorylation of p65 at Serine 536. As evident from Figure 2, the anti-P-p65 Ser536 antibody recognizes a $65 \mathrm{kDa}$ immunoreactivity (B1) induced by TNF- $\alpha$, that comigrates with unphosphorylated p65 (Figure 2, B2) and is indeed inhibited when p65 is silenced. Equal loading of the gels was confirmed by detection of tubulin (A3, B3).
3.3. Other Anti-Phospho-p65 Ser276 Antibodies Display the Same Immunoreactivity as the Cell Signaling no. 3037 Antibody. The Bands Detected by All Presumable P-p65Ser276-Specific Antibodies Are Not p65, But Unrelated PKARegulated Proteins. To investigate whether other available antibodies would perhaps be more useful to detect Ser276 phosphorylated p65 via Western Blotting, we investigated phosphorylation of p65 at Ser276 using two anti-P-p65 Ser276 antibodies distributed by independent companies (no. 11011 from SAB and no. 100-401-264 from Rockland). In addition, we used a homemade antibody (kind gift of Prof. P. Cohen, University of Dundee, UK) that was previously used to demonstrate Ser276 phosphorylation of p65 (10). 
As apparent from Figure 3, like Cell Signaling no. 3037 (B3), both SAB no. 11011 (A1) and Rockland no. 100401-264 (B1) and the homemade antibody (C1) detect a major $130 \mathrm{kDa}$ band (upper arrow), in isoproterenol-treated cells. The SAB no. 11011 and homemade antibodies also detect the $80 \mathrm{kDa}$ inducible band (lower arrow), whereas this band was not apparent with the Rockland no. 100-401264 antibody. The SAB no. 11011 and homemade antibodies in addition recognize several high molecular weight bands, some of which are induced by isoproterenol. Whereas siRNAmediated silencing clearly reduced the p65 content of the cells (A2, B2, C2), none of the bands detected by the four anti-P-p65 Ser276 antibodies was concomitantly reduced (A1, B1, B3, C1).

The p65 Ser276 residue is part of a consensus recognition site (RRXS) for PKA [15]. To investigate whether perhaps the anti-P-p65 Ser276 antibodies crossreact with other PKAregulated proteins, we investigated what happens to the antiP-p65 Ser276-reactive bands upon silencing PKAc $\alpha$. As evident from Figure 3 (A1, B1, B3), the isoproterenol-induced bands detected by all four anti-P-p65 Ser276 antibodies were almost completely inhibited in cells where PKAc $\alpha$ was (partially) silenced (A2, B2). Equal protein loading on the gels was confirmed by detection of the blots with anti-tubulin (Supplementary Figure 1) (the image in B3 was obtained upon stripping of the B blot after tubulin detection and reprobing with Cell Signaling no. 3037; knock-down and tubulin controls are, hence, the same as for B1).

3.4. Cell Signaling no. 3037 and SAB no. 11011 Do Not Detect Any Additional Immunoreactive Bands in $065-1-$ MEF Cells, Reconstituted with Wild Type p65, That Are Not Apparent in p65 -/- MEF Cells. Whereas the siRNA-mediated knockdown of p 65 was very efficient in the $1321 \mathrm{~N} 1$ cells we used in this study, knock-down is never $100 \%$ complete. Therefore, we compared the immunoreactivity of the Cell Signaling no. 3037 and SAB no. 11011 antibodies in Western Blots from p65 knock-out MEF cells and MEF cells that had been reconstituted with either wild type p65 (p65 wt) or p65 with Ser276 mutated to alanine (p65 S/A). As apparent from Figure 4, the typical immunoreactive pattern (a prominent $130 \mathrm{kDa}$ and a weak $80 \mathrm{kDa}$ ) band is also present in MEF cells that are deficient of p65. Unlike in the other investigated cell types, these bands seem to be present constitutively in MEF cells. In addition a $50 \mathrm{kDa}$ band is induced by forskolin. Most importantly, not one single band is detected by either the Cell Signaling no. 3037 and SAB no. 11011 antibodies in p65 wt reconstituted MEF cells, that is not already present in p65 knock-out cells (Figure 4, A1 and B1), which clearly have no p65 (Figure 4, A2 and B2).

3.5. The Detected $80 \mathrm{kDa}$ and $130 \mathrm{kDa}$ Immunoreactivities Are Not p105/p50 or c-Rel. In the datasheet of the Cell Signaling no. 3037 antibody, it is mentioned that the antibody might crossreact with c-Rel and NF- $\kappa$ B1 (p105/p50) when phosphorylated at the homologous Ser267 and Ser337 residues, respectively. As $c-R e l$ and NF- $\kappa$ B1 p105 have molecular weights of 70 and $105 \mathrm{kDa}$, respectively, upon phosphorylation they could theoretically appear as the 80 and $130 \mathrm{kDa}$ bands we observed on Western Blot. We therefore investigated whether siRNA-mediated silencing of p105/p50 or c-Rel affected the appearance of these bands on Western Blot. As apparent from Figure 5, whereas we were able to efficiently inhibit the expression of c-Rel and p105, the intensity of the 80 and $130 \mathrm{kDa}$ immunoreactive bands was unaltered, indicating they do not represent phosphorylated p105/p50 or c-Rel.

3.6. The Cell Signaling no. 3037 Antibody Recognizes p65 Ser276 Phosphorylation In Vitro. As the above described results indicate that the Cell Signaling no. 3037 antibody (as well as the other anti-P-p65 Ser276 antibodies we tested) does not allow detection of in vivo Ser276 phosphorylation of p65 via Western Blotting, we next assessed whether the Cell Signaling no. 3037 antibody recognized in vitro Ser276phosphorylated p65. We therefore performed an in vitro kinase assay, using recombinant MSK-1 to phosphorylate a recombinant GST-p65 fusion protein. As demonstrated in Figure 6, we could indeed detect phosphorylation of GSTp65, that was blocked by preadsorption with the immunizing phosphopeptide (see dot blot), and that was inhibited by inclusion of the PKA/MSK-1 inhibitor H89 in the kinase reaction (see Western Blot). When a GST-p65 Ser276 to cysteine mutant (SC) was used in the kinase reaction, no immunoreactivity could be observed neither in the dot blot nor in the Western Blot, underlining the specificity of the recognition. These results indicate that, in vitro, the Cell Signaling anti-P-p65 Ser276 antibody recognizes Ser276phosphorylated p65.

\section{Discussion}

Here we show that, several commonly used antibodies, designed to detect Ser276-phosphorylated p65, react with other PKA-regulated proteins, but do not detect Ser276phosphorylated p65 in vivo via Western Blotting.

According to the manufacturer's data sheet, the Ser276phosphorylated p65 band detected by the Cell Signaling no. 3037 antibody migrates at $80 \mathrm{kDa}$ in denaturing gels. We indeed detected a weak $80 \mathrm{kDa}$ band, that appears to be coregulated with a much more prominent $130 \mathrm{kDa}$ band, in a variety of mouse and human cell types (L929sA, Raw264.7, C2C12, MEF cells, L363, A549, and 1321N1 cells) and upon treatment with different known inducers (LPS, PMA, TNF, forskolin, cAMP) of p65 Ser276 phosphorylation. In some cell types, visualization of the $80 \mathrm{kDa}$ band required more sensitive fluorescent (Odyssey Imaging System, Licor, Lincoln, NA) development procedures than the standard HRPbased chemiluminescent detection we are routinely using. The 80 and $130 \mathrm{kDa}$ bands disappeared upon preadsorption of the phosphospecific antibody with the phosphopeptide that was used for immunization, indicating they represent bands that are specifically recognized by the antibody.

We further investigated Ser276 phosphorylation of p65 mainly in the human $1321 \mathrm{~N} 1$ astrocytoma cell line, upon treatment with the $\beta$-adrenergic agonist isoproterenol (to 


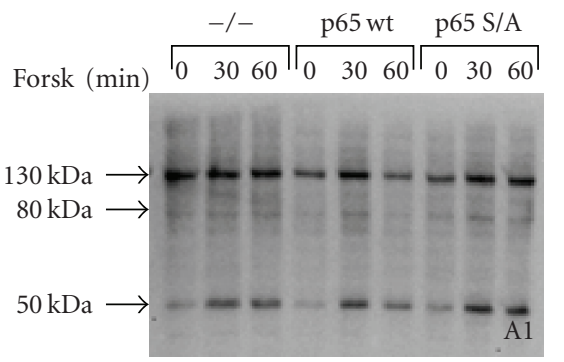

ID:anti-P-p65 ser 276 cell signaling \# 3037

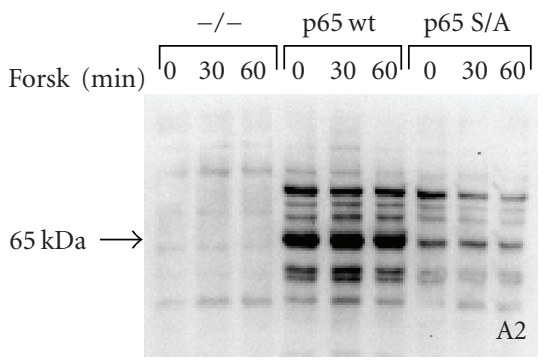

ID: anti-p65

(a)

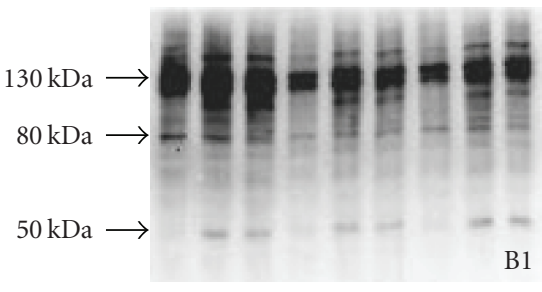

ID:anti-P-p65 ser 276 SAB \# 11011

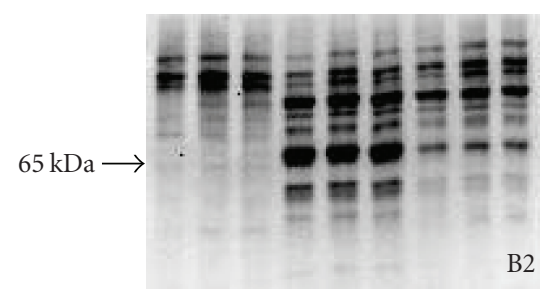

ID: anti-p65

(b)

Figure 4: Presence of anti-P-p65 Ser276-reactive bands in p65 deficient MEF cells. p65 knock-out MEF cells (-/-), or p65 -/- MEF cells reconstituted with wild type p65 (p65 wt) or p65 with Ser276 mutated to alanine (p65 S/A), were induced with forskolin (forsk, $10 \mu \mathrm{M})$ for 0, 30, and $60 \mathrm{~min}$. Presence of Ser276-phosphorylated p65 in cell lysates was analysed by Western Blot using Cell Signaling no. 3037 (A1) or SAB no. 11011 (B1). Blots were extensively washed and reprobed with anti-p65 to confirm p65 absence in -/- MEFs, and p65 presence in wt and S/A reconstituted MEFs (A2 and B2, arrows indicate anti-p65 detected bands).

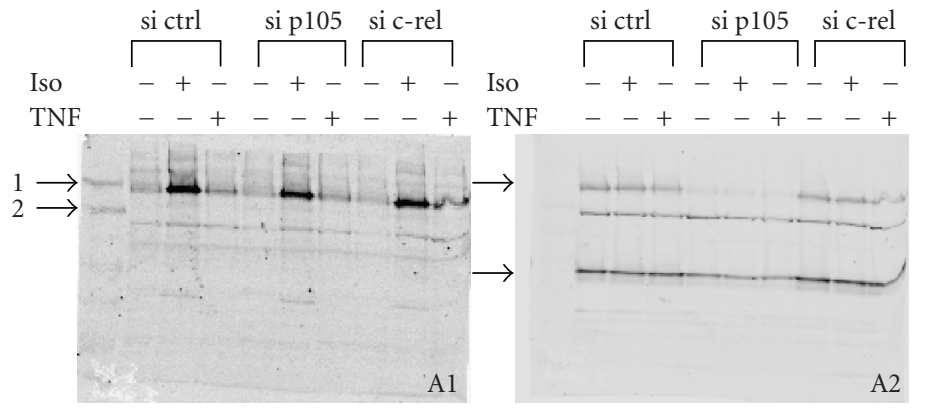

ID: anti-P-p65 ser276

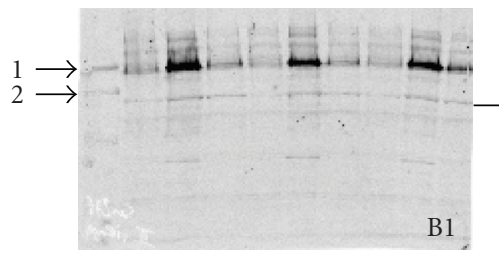

ID: anti-P-p65 ser276
ID: anti-p105/p50

(a)

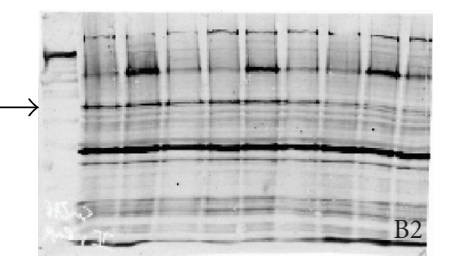

ID: anti-c-rel

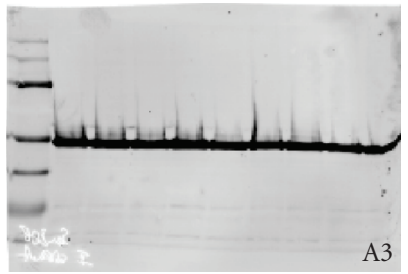

ID: anti-tubulin

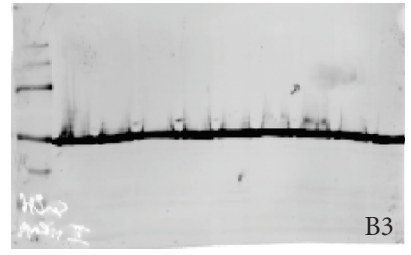

ID: anti-tubulin

(b)

Figure 5: The detected $130 \mathrm{kDa}$ and $80 \mathrm{kDa}$ immunoreactivities are not NF- $\kappa B$ p105/p50 or c-Rel. $1321 \mathrm{~N} 1$ cells were transiently transfected with control, p105/p50 or c-Rel siRNA as described in Section 2. Cells were induced for 30 min with TNF- $\alpha(2000 \mathrm{IU} / \mathrm{mL})$ or iso (10 $\mu \mathrm{M})$. Presence of Ser276-phosphorylated p65 in cell lysates was analysed by Western Blot using Cell Signaling no. 3037 (A1, B1). 130 and 80 kDa immunoreactivities are indicated by arrows ( 1 and 2 resp.). Because of the overlap of the $130 \mathrm{kDa}$ and the p105 immunoreactive bands, blot A was first stripped and reprobed with anti-p105/p50 (A2). p105 immunoreactivity is indicated by the upper arrow; p50 immunoreactivity is indicated by the lower arrow. Blot B was extensively washed and reprobed (without stripping) with anti-c-Rel (B2). c-Rel immunoreactivity is indicated by an arrow. Tubulin was used as a loading control (A3, B3). 


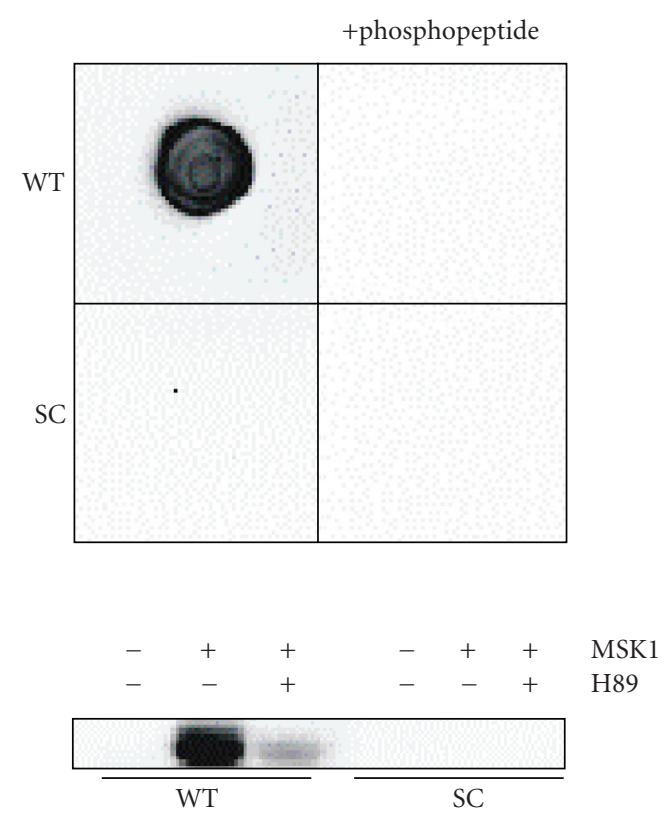

FIGURE 6: In vitro recognition of the phosphorylated Serine 276 residue of p65 by Cell Signaling no. 3037. Recombinant wt p65-GST or SC mutant p65-GST fusion proteins were in vitro phosphorylated using recombinant MSK-1. $250 \mathrm{ng}$ of the recombinant proteins were spotted on a nitrocellulose membrane and subjected to Western Blotting with Ser276 phospho-specific p65 antibody, either preincubated with $10 \mu \mathrm{g} / \mathrm{mL}$ phosphorylated peptide that was used to generate the antibody (upper panel) or not. $1 \mu \mathrm{g}$ of recombinant protein was separated by SDS-PAGE after the in vitro kinase assays performed either in the presence of active MSK-1 and/or $10 \mu \mathrm{M}$ H89 (lower panel) or not.

activate the PKA pathway) and with TNF- $\alpha$ (to activate MSK1). In Western Blotting experiments, upon isoproterenol treatment, the $1321 \mathrm{~N} 1$ cells exhibit the typical immunoreactive bands that are also detected by Cell Signaling no. 3037 in other cell types and upon different treatments: a very prominent $130 \mathrm{kDa}$ band and a very weak $80 \mathrm{kDa}$ band, which was also slightly induced by TNF- $\alpha$. Our observation that all immunoreactive bands persisted upon siRNA-mediated silencing of p65 in $1321 \mathrm{~N} 1$ cells, however, indicates that these bands are not posttranslationally modified forms of p65 (Figure 2). The successfulness of the siRNA approach to knock-out p65 was corroborated by the finding that silencing of p65 did inhibit TNF- $\alpha$-induced p65 Ser536 phosphorylation (Figure 2). In line with these findings, using Cell Signaling anti-P-p65 Ser276, we were unable to detect the $130 \mathrm{kDa}$ and $80 \mathrm{kDa}$ immunoreactivities on Western Blot upon successful immunoprecipitation of endogenous p65 in lysates from isoproterenol-treated $1321 \mathrm{~N} 1$ cells, whereas we did successfully recover Ser536-phoshorylated p65 (Supplementary Figure 2). In addition, we were also unable to detect any anti-P-p65 Ser276 immunoreactive bands when Flag-tagged p 65 was transiently transfected and subsequently concentrated by anti-Flag immunoprecipitation (Supplementary Figure 3). We could however successfully detect
Ser276-phosphorylated p65 with the Cell Signaling anti-Pp65 Ser276 using an in vitro kinase approach (Figure 6). These observations indicate that the Cell Signaling anti-Pp65 Ser276 antibody does specifically recognize the phosphorylated p65 Ser276 residue when p65 is present abundantly, and isolated from other possible crossreacting proteins, as is the case in the in vitro assay. However, in an in vivo situation, when $\mathrm{p} 65$ is present in a mixture of other proteins with a homologous phosphorylation sequence, the antibody clearly has a preference for other targets, that are either more abundant, are more efficiently phosphorylated, or have a higher affinity for the anti-P-p65 Ser276 antibodies than Ser276-phosphorylated $\mathrm{p} 65$. As we had identical results using different lot numbers of Cell Signaling no. 3037, as well as using anti-P-p65 Ser276 antibodies distributed by different companies (SAB no. 11011 and Rockland no. 100-401-264) and a homemade antibody (Figure 3), we believe our results are not artefactual. All investigated antibodies were probably generated with very similar synthetic phosphopeptides (i.e., MQLRRPPSDRELSE for the homemade antibody and QLRRPpSDRELSE for the Rockland no. 100-401-264 antibody). The sequence surrounding the $\mathrm{p} 65$ Ser276 residue resembles a PKA recognition site [15] and our observation, that siRNAmediated silencing of PKAc $\alpha$ leads to disappearance of the anti-P-p65 Ser276-detected immunoreactivities (Figure 3), indicates the antibodies indeed detect PKA-phosphorylated proteins, but unfortunately not PKA-phosphorylated p65, in $1321 \mathrm{~N} 1$ cells. The fact that we were unable to immunoprecipitate the inducible 80 or $130 \mathrm{kDa}$ proteins using anti-Pp65 Ser276 (data not shown), hampers identification of the crossreacting proteins via mass spectrometric analysis.

Although our data indicate that in the $1321 \mathrm{~N} 1$ cell line, the anti-P-p65 Ser276 antibodies we investigated do not detect Ser276-phosphorylated p65, but rather crossreact with other PKA-regulated proteins, we cannot claim, just by analogy, that the bands, recognized by these antibodies in other cell types, are neither Ser276-phosphorylated p65. We did, however, obtain uncircumstantial evidence that at least the Cell Signaling no. 3037 and SAB no. 11011 antibodies lack specificity for $\mathrm{p} 65$, by the observation that in lysates from p65-deficient MEF cells, and from p65 MEF cells reconstituted with wild type p65, the same immunoreactive bands are detected (Figure 4). In addition, in MEF cells, as in all other investigated cell types, constitutive immunoreactivities of $130 \mathrm{kDa}$ and $80 \mathrm{kDa}$ were apparent, indicating these bands probably represent crossreacting proteins that are abundantly present in most cell types.

Phosphorylation of p65 at the Ser276 residue has been extensively investigated in other cell types than the ones we have studied, yet several groups have demonstrated phosphorylation of p65 Ser276 via Western Blot in cells that we also investigated (Supplementary Table 1), without reporting any peculiarities on the MW of the bands shown in the Western Blots. Only in a few of these publications, additional, antibody-independent, experiments were performed that support the involvement of p65 Ser276 phosphorylation and the conclusion of their studies.

The main conclusion from this study is that reports on the function of Ser276 phosphorylation of p65 should 
include additional, antibody-independent experiments. This, however, narrows down p65 phosphorylation research to the study of p65 -/- cells, reconstituted with selected p65 point mutants. Very recently, the group that originally reported Ser276 phosphorylation by PKA [9], and, as a matter of fact, has never used the anti-P-p65-antibody approach, has generated a transgenic knock-in mouse with p65 Ser276 mutated to alanine [12]. Still, for those interested in studying p65 phosphorylation in vivo in cells and tissues, phosphospecific antibodies are invaluable tools. We therefore believe it might be worthwhile to invest in the generation of a monoclonal anti-P-p65 Ser276 antibody, with a better specificity and selectivity profile than the currently available polyclonal antibodies.

In summary, we believe the take-home-message from our investigation is that caution is warranted in the interpretation of data generated using the abovedescribed antiphospho-p65Ser276 antibodies.

\section{Acknowledgments}

The authors would like to thank all authors from whom they received feedback on their experience with the tested anti-P-p65Ser276 antibodies. They would also like to thank Professor P. Cohen for the kind gift of the homemade antiP-p65Ser276 antibody. This work was supported by the FWO flanders. A. Spooren is a predoctoral FWO-fellow. L. Vermeulen and S. Gerlo are post-doctoral FWO-fellows. Financial support was also provided by Interuniversity Attraction Poles (IAP) P5/12 and by an institutional grant (GOA) from Ghent University.

\section{References}

[1] H. Singh, R. Sen, D. Baltimore, and P. A. Sharp, "A nuclear factor that binds to a conserved sequence motif in transcriptional control elements of immunoglobulin genes," Nature, vol. 319, no. 6049, pp. 154-158, 1986.

[2] M. S. Hayden and S. Ghosh, "Shared principles in NF- $\kappa \mathrm{B}$ signaling," Cell, vol. 132, no. 3, pp. 344-362, 2008.

[3] S. Ghosh and M. S. Hayden, "New regulators of NF- $\kappa$ B in inflammation," Nature Reviews Immunology, vol. 8, no. 11, pp. 837-848, 2008.

[4] P. Viatour, M.-P. Merville, V. Bours, and A. Chariot, "Phosphorylation of NF- $\kappa \mathrm{B}$ and $\mathrm{I} \kappa \mathrm{B}$ proteins: implications in cancer and inflammation," Trends in Biochemical Sciences, vol. 30, no. 1, pp. 43-52, 2005.

[5] L. Vermeulen, W. Vanden Berghe, and G. Haegeman, "Regulation of NF- $\kappa \mathrm{B}$ transcriptional activity," Cancer Treatment and Research, vol. 130, pp. 89-102, 2006.

[6] M. L. Schmitz, I. Mattioli, H. Buss, and M. Kracht, "NF- $\kappa$ B: a multifaceted transcription factor regulated at several levels," ChemBioChem, vol. 5, no. 10, pp. 1348-1358, 2004.

[7] L.-F. Chen and W. C. Greene, "Shaping the nuclear action of NF- $\kappa \mathrm{B}$," Nature Reviews Molecular Cell Biology, vol. 5, no. 5, pp. 392-401, 2004.

[8] M. Neumann and M. Naumann, "Beyond I $\kappa$ Bs: alternative regulation of NF- $\kappa$ B activity," FASEB Journal, vol. 21, no. 11, pp. 2642-2654, 2007.

[9] H. Zhong, H. SuYang, H. Erdjument-Bromage, P. Tempst, and
S. Ghosh, "The transcriptional activity of NF- $\kappa \mathrm{B}$ is regulated by the I $\kappa$ B-associated PKAc subunit through a cyclic AMPindependent mechanism," Cell, vol. 89, no. 3, pp. 413-424, 1997.

[10] L. Vermeulen, G. De Wilde, P. Van Damme, W. Vanden Berghe, and G. Haegeman, "Transcriptional activation of the NF- $\kappa$ B p 65 subunit by mitogen- and stress-activated protein kinase-1 (MSK1)," EMBO Journal, vol. 22, no. 6, pp. 13131324, 2003.

[11] H. Zhong, R. E. Voll, and S. Ghosh, "Phosphorylation of NF- $\kappa$ B p65 by PKA stimulates transcriptional activity by promoting a novel bivalent interaction with the coactivator CBP/p300," Molecular Cell, vol. 1, no. 5, pp. 661-671, 1998.

[12] J. Dong, E. Jimi, H. Zhong, M. S. Hayden, and S. Ghosh, "Repression of gene expression by unphosphorylated NF- $\kappa \mathrm{B}$ p65 through epigenetic mechanisms," Genes and Development, vol. 22, no. 9, pp. 1159-1173, 2008.

[13] B. Vanhaesebroeck, S. Van Bladel, A. Lenaerts, et al., "Two discrete types of tumor necrosis factor-resistant cells derived from the same cell line," Cancer Research, vol. 51, no. 9, pp. 2469-2477, 1991.

[14] C. Yoon, Z. Korade, and B. D. Carter, "Protein kinase Ainduced phosphorylation of the p65 subunit of nuclear factor$\kappa \mathrm{B}$ promotes Schwann cell differentiation into a myelinating phenotype," The Journal of Neuroscience, vol. 28, no. 14, pp. 3738-3746, 2008.

[15] G. Mosialos and T. D. Gilmore, "v-Rel and c-Rel are differentially affected by mutations at a consensus protein kinase recognition sequence," Oncogene, vol. 8, no. 3, pp. 721-730, 1993. 

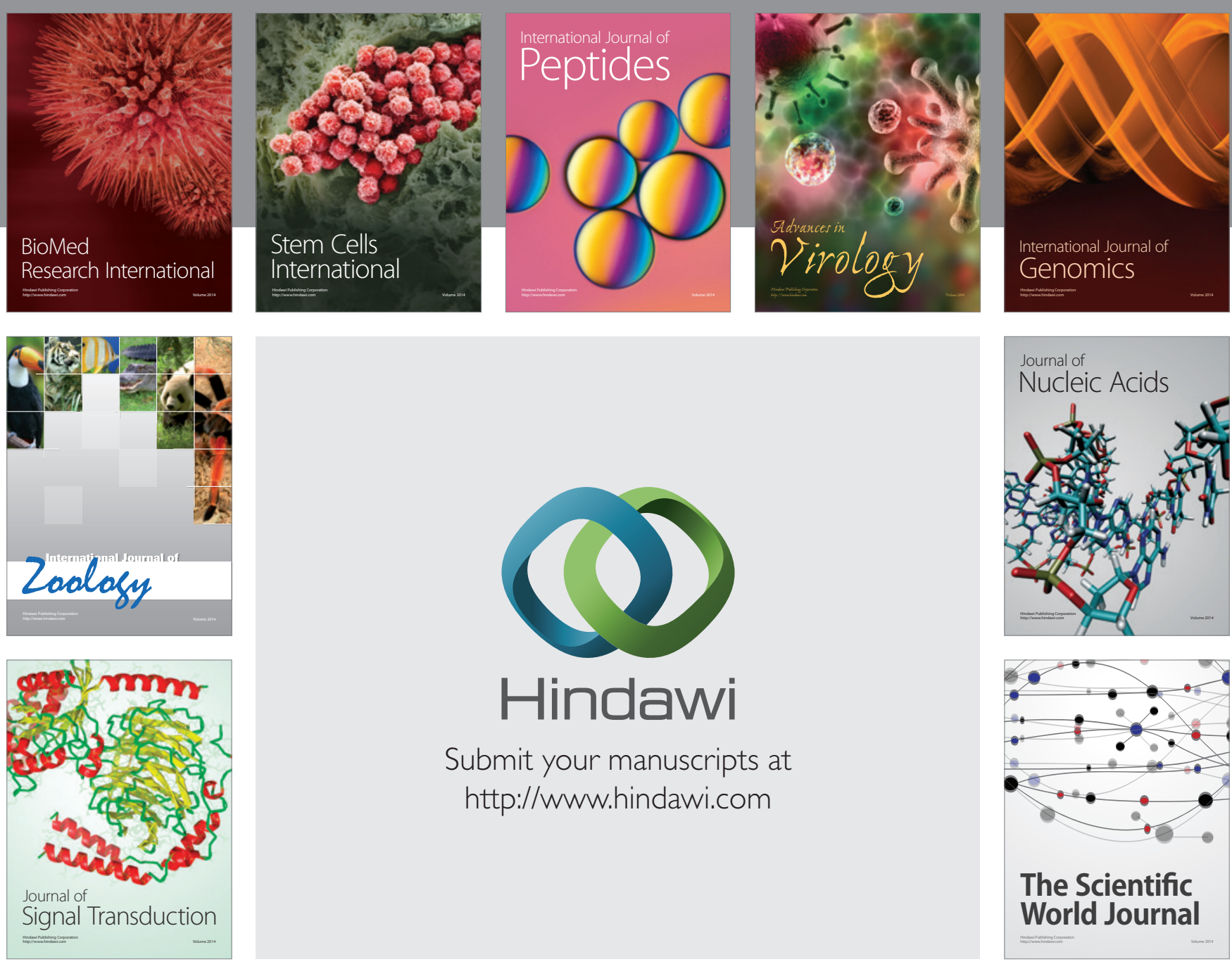

Submit your manuscripts at

http://www.hindawi.com
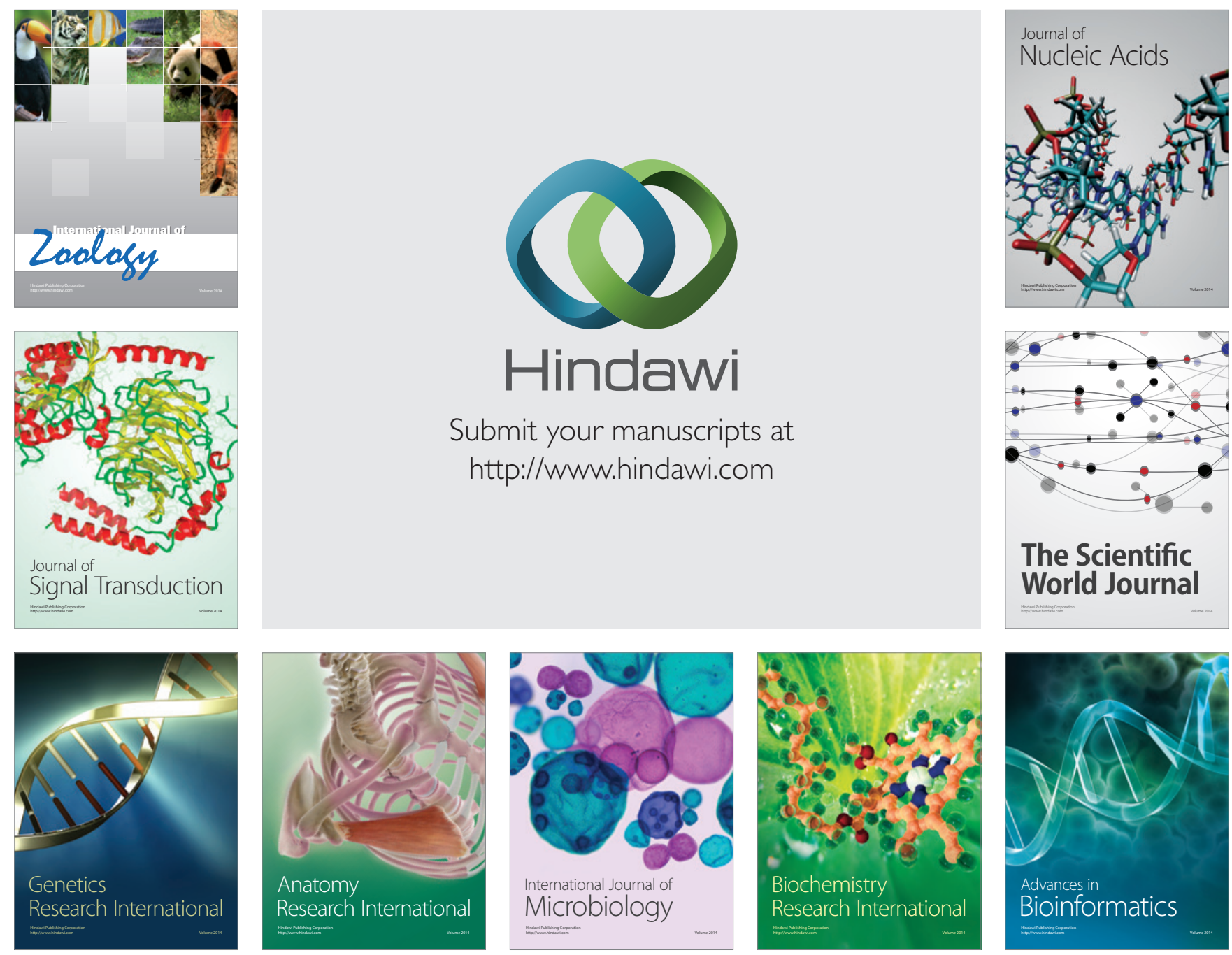

The Scientific World Journal
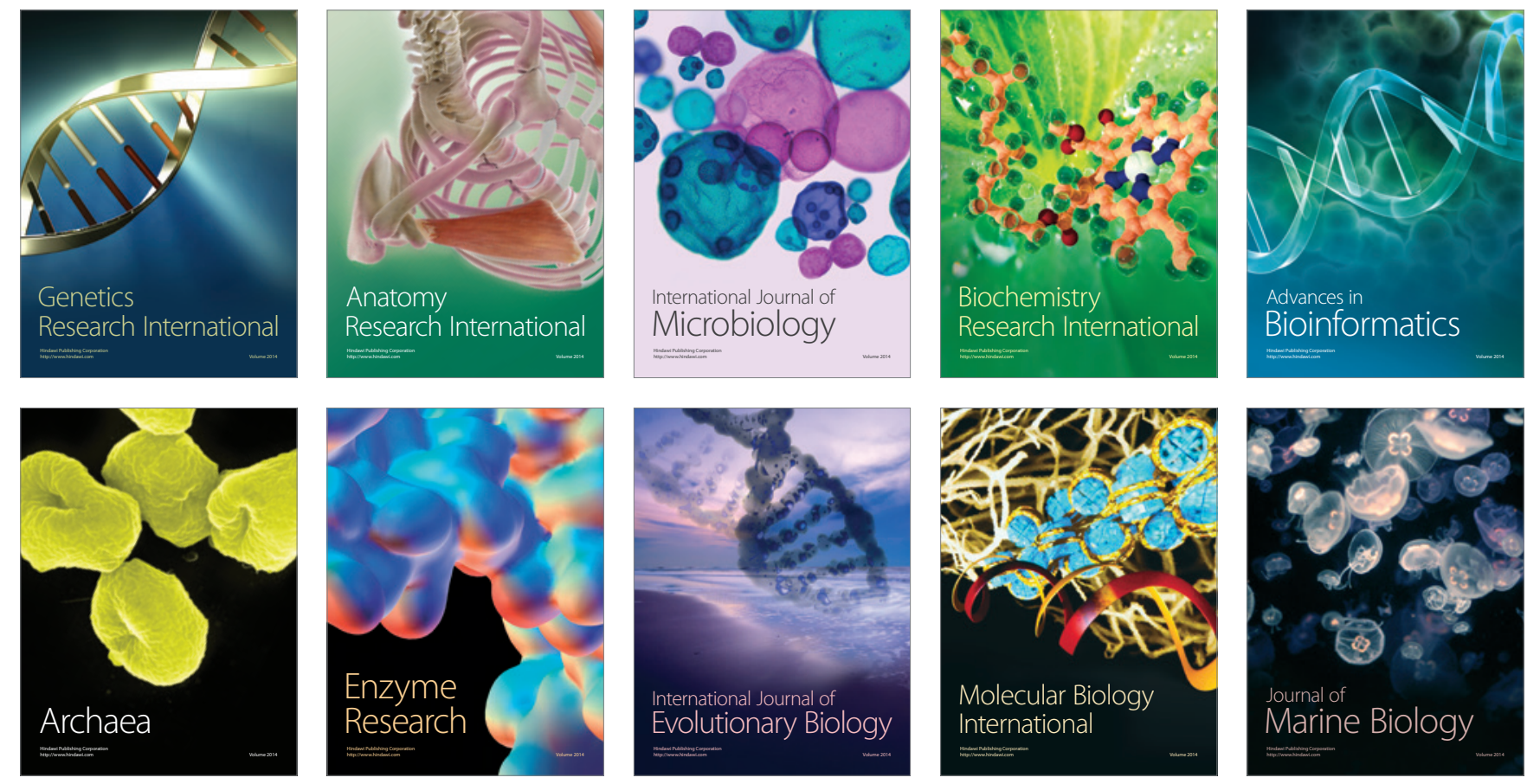